\title{
On the propagation of firing rate and synchrony in a model of cortical network
}

\author{
Arvind Kumar*1,2,4, Stefan Rotter ${ }^{2,3}$ and Ad Aertsen ${ }^{1,2}$
}

\author{
Address: ${ }^{1}$ Neurobiology \& Biophysics, Faculty of Biology, University of Freiburg, Germany, ${ }^{2}$ Bernstein Center for Computational Neuroscience \\ Freiburg, Germany, ${ }^{3}$ Theory \& Data Analysis, Institute for Frontier Areas of Psychology and Mental Health Freiburg, Germany and ${ }^{4}$ Current \\ address: Department of Neuroscience, Brown University, Providence, RI, USA \\ Email: Arvind Kumar* - arvind_kumar@brown.edu \\ * Corresponding author
}

from Seventeenth Annual Computational Neuroscience Meeting: CNS*2008

Portland, OR, USA. 19-24 July 2008

Published: II July 2008

BMC Neuroscience 2008, 9(Suppl I):P6 doi:10.1 I86/I47I-2202-9-SI-P6

This abstract is available from: http://www.biomedcentral.com/I47I-2202/9/SI/P6

(C) 2008 Kumar et al; licensee BioMed Central Ltd.

Sensory information in the brain is processed in multiple stages. In this view of information processing, the information needs to be carried from one cortical region or area to the next. A very simple model for this type of processing is a feed forward chain of groups of neurons (FFN), in which each neuron in a given group receives multiple synaptic inputs from neurons in the previous group [1]. FFNs were shown to have different operating modes, where they transmit either firing rates or synchronous volleys of spikes [2-4]. These findings have been partially confirmed in in vitro experiments [5]. The transmission of information in a FFN, be it rate based or synchrony based, is strongly influenced by background activity $[2,4]$. In most studies of isolated FFNs the background was assumed to be independent of the FFN activity. In a more realistic scenario, where the FFN is embedded into a recurrent cortical network, the activity of the FFN interacts with the background activity in the network. In fact, it was shown that embedding non-random structures with a high degree of shared connectivity, such as an FFN, may introduce instabilities in the network dynamics, such that excitation of only a small group of neurons would be sufficient to induce synchronization of the activity of the entire network [6]. Is this instability a generic property of random networks or an artifact of a too simple model? Recent theoretical work has suggested that conductance-based synapses may help stabilizing the dynamics of the network [7]. This suggestion was motivated by the fact that in networks in a high activity regime, post-synaptic-potentials are strongly attenuated due to the high conductance state. Here, we embedded an FFN in a locally-connected random network. We show that by modeling synapses as conductance transients, rather than current sources, it becomes possible to embed and propagate transient synchrony in the FFN, without destabilizing the background network activity. However, the network activity has a strong impact on the conditions under which propagation of activity in the embedded FFN is possible. Global synchrony and high firing rates in the embedding network prohibit the propagation of both synchronous and asynchronous spiking activity. By contrast, asynchronous low rate network states support the propagation of synchronous spiking and asynchronous, albeit only low, firing rates [8]. In either case, spiking activity tends to synchronize as it propagates, rendering the transmission of information only in firing rates problematic. Finally, asynchronous background activity allows us to embed more than one FFN, with the amount of cross-talk depending on the degree of overlap in the FFNs, opening the possibility of computational mechanisms utilizing transient synchrony among the activities in multiple FFNs.

\section{Acknowledgements}

This work was supported by the German Federal Ministry of Education and Research (BMBF grant 0IGQ0420 to BCCN Freiburg), GIF, DFG-GraKo (no. 843) and the 6th RFP of the EU (grant no. I5879-FACETS).

\section{References}

I. Abeles M: Corticonics: Neural circuits of the cerebral cortex Cambridge: Cambridge U Press; 1991. 
2. Diesmann M, Gewaltif M-O, Aerstsen A: Stable propagation of synchronous spiking in cortical neural networks. Nature 1999, 402:529-533.

3. Litvak V, Sompolinsky H, Segev I, Abeles M: On the transmission of rate code in long feed-forward networks with excitatoryinhibitory balance. J Neurosci 2003, 23:3006-30I5.

4. van Rossum MCW, Turrigiano GG, Nelson SB: Fast propagation of firing rates through layered networks of noisy neurons. J Neurosci 2002, 22(5): 1956-1966.

5. Reyes AD: Synchrony-dependent propagation of firing rate initeratively constructed networks in vitro. Nat Neurosci 2003 , 6:593-599.

6. Mehring C, Hehl U, Kubo M, Diesmann M, Aertsen A: Activity dynamics and propagation of synchronous spiking in locally connected random networks. Biol Cybern 2003, 88:395-408.

7. Kuhn A, Aertsen A, Rotter S: Neuronal integration of synaptic input in the fluctuation-driven regime. J Neurosci 2004, 24:2345-2356.

8. Kumar A, Rotter S, Aertsen A: Stable propagation of synchronized spiking in locally connected random networks. J Neurosci 2008.

Publish with Bio Med Central and every scientist can read your work free of charge

"BioMed Central will be the most significant development for disseminating the results of biomedical research in our lifetime. "

Sir Paul Nurse, Cancer Research UK

Your research papers will be:

- available free of charge to the entire biomedical community

- peer reviewed and published immediately upon acceptance

- cited in PubMed and archived on PubMed Central

- yours - you keep the copyright 